\title{
The role of intelligent habitats in upholding elders in residence
}

\author{
H. Pigot ${ }^{1}$, B. Lefebvre ${ }^{2}, J-G$. Meunier $^{2}$, B. Kerhervé ${ }^{2}$, A. Mayers ${ }^{1}$ \\ \& S. Giroux ${ }^{1}$ \\ ${ }^{\prime}$ Département de mathématiques et d'informatique, Université de \\ Sherbrooke, Canada \\ ${ }^{2}$ Département d'informatique, Université du Québec à Montréal, Canada
}

\begin{abstract}
The intelligent habitat is made of fixed components (movements detectors and intelligent electric household appliances) and small mobile processors worn by the elder. Fixed and mobile components communicate to assist the elder in performing his tasks and to intervene in case of risk. The system has two types of features: those carried out inside the residence (information acquisition, cognitive help like sound or visual cues when everyday life activity is carried out in an incomplete or dangerous way) and those reporting to the relatives and the external care network major risk events or evolution of the elder health state. The system intervention with the elder must be personalized according to the incurred risk gravity, his health state, his life habits and his preferred interaction mode: image, text, sound, voice...
\end{abstract}

\section{Introduction}

Frail elders are suffering from several chronic diseases. They legitimately wish to remain at home as long as possible. For economic reasons, governments also want to maintain them in their residence. However this brings safety problems. The incurred risks can be classified in two 
categories: immediate risks (falls, fire, inappropriate drug ingestion) and long-term risks (bad diet, deficient hygiene). Thanks to the recent striking progress and technology convergence - devices, networks and artificial intelligence-, the habitat may not be any more passive but it may become active and intelligent to assist elders in their daily activities and to inform relatives and caregivers as soon as necessary.

This paper sets the theoretical and practical framework for risk minimization through actions undertaken by the elder physical environment. More precisely the focus is on the elders' houses. First we sketch the required computer infrastructure. The computer infrastructure of an intelligent habitat is divided in three layers. The application layer, the upper one, offers services of telemonitoring, task support and interaction with the outer world $(\$ 4)$. The hardware layer, the lower one, contains sensors, electrical devices, and so on (\$2). It collects information and transmits it toward the superior layer. However given the diversity, the heterogeneity and the low-level nature of hardware, it is necessary to add an intermediate layer that permits to bind applications to the hardware. This layer serves as the middleware and contains various frameworks $(\$ 3)$. Next we describe the compulsory models to support intelligent behaviour and rational decision making. These models are the model of the person ( $\$ 5)$, the model of the tasks $(\S 6)$ and the model of the environment (\$7). Finally we depict the modules doing interventions : telemonitoring (externally oriented) and cognitive assistance (internally oriented) $(\S 8)$.

\section{The hardware layer}

The hardware layer relies on an experimental Smart Home which has been conceived at the Grenoble Faculty of Medicine by the AFIRM team of the TIMC-IMAG laboratory (University J. Fourier, Grenoble, France) [11]. This smart home is equipped in order to monitor the activity and the status of a person at home [11].

Four categories of sensors support the monitoring process: activity, actimetry, physiology and environment. Activity sensors enable to track people movements from room to room. They are either infrared-based devices or magnetic contact switches. Actimetry sensors are used to detect fall, vibration... The body actimetry sensor is a wearable sensor which has been developed to predict the situation of a person. It is composed of three sensors: vertical acceleration, body orientation and mechanical vibration of the body surface. The combination of data given by activity sensors and actimetry sensors is used to determine the position of the person, for instance sleeping, lying after a fall, walking... Physiology sensors collect 
physiological parameters such as blood pressure or weight. Environment sensors detect smoke, measure home temperature and hygrometry, etc.

In the Grenoble implementation, all these sensors are connected, wirelessly and through a CANTM bus, to a software agent hosted on a PC at the patient's home to perform signals analysis and detection of critical situations. In case of critical situation, this agent can communicate, via Internet, with a Telecare Control Centre responsible for collecting and interpreting alerts, and transferring the appropriate messages to the people concerned [10].

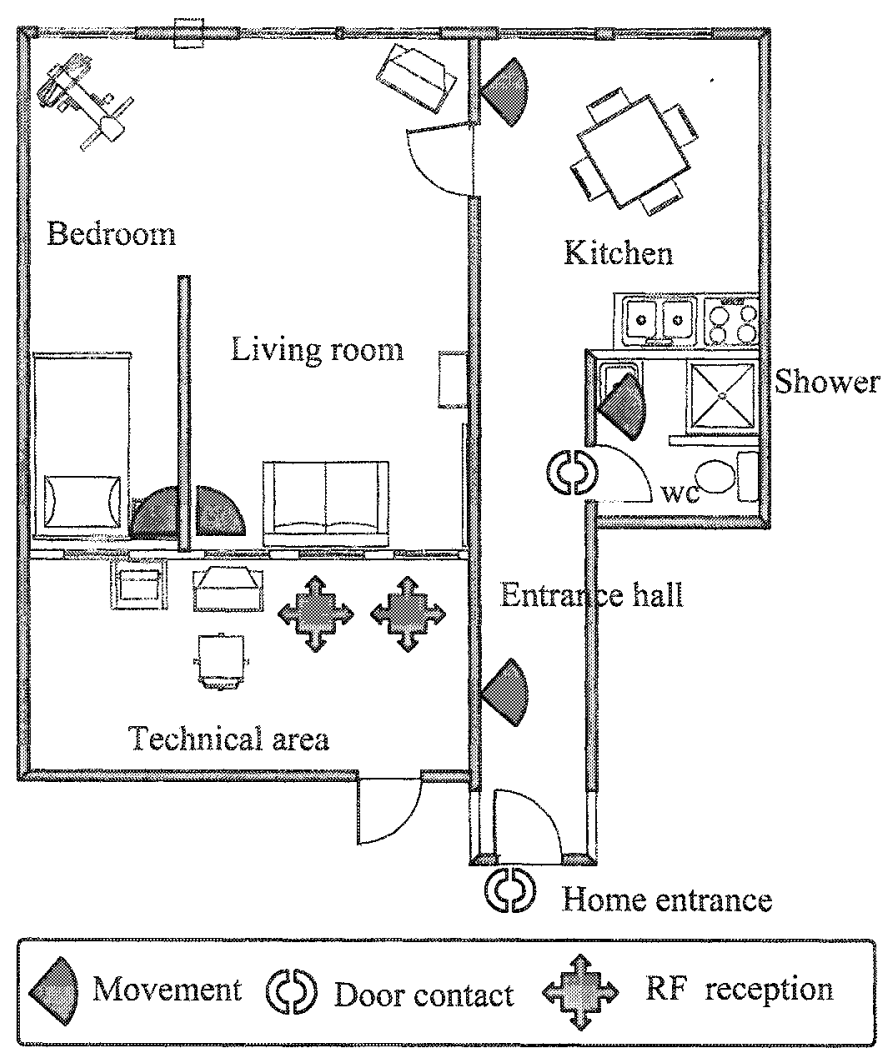

Figure 1: The Grenoble Experimental Smart Home. 


\section{The middleware layer}

The hardware layer is thus responsible for gathering raw numeric data and to forward them to the application layer. The latter then analyses and merges numeric data generally to produce symbolic values that are stored in the models. This symbolic representation is then used to reason and to take actions. This pattern of organisation may seem easy and quite straightforward to implement. Unfortunately the computing infrastructure of a real intelligent habitat must cope with constraints that render far more complex to handle its implementation than it may be thought as first sight. As a result the overall resulting infrastructure must provide support for wireless and spontaneous networks, reflective component, distributed systems and distributed algorithms.

Indeed, cables may rapidly become a nightmare in maintaining and evolving an intelligent habitat as devices may be moved, or new devices may be added... On the other hand, wearable devices must be able to join to the system when they enter the habitat. Obviously most part of devices should connect wirelessly. For similar reasons, networking should be spontaneous. The addition, the removal, the failure or the modification of a component must be handled without human intervention.

As technology evolves, new kinds of devices may be added; it is not practical to need to reprogram the system each time a new device category or new software features become available. Hence each component, be it a device or a piece of software, must be self-describing to enable cooperation with the other components. Using their reflective facilities, components will also join more easily to spontaneous networks.

Finally the underlying system is clearly distributed over devices and software components. Each component must have at least a partial autonomy to take local decision. If there is fire on the stove, the local component should not refer to a centralized entity that reasons and eventually decides to shut down the stove element. This decision should be taken locally. As a consequence, reasoning algorithms are also distributed over devices and software components and code may move across devices. Complexity of each autonomous component is then reduced while local decisions and interactions between components confer to the system a globally intelligent behaviour. Given the peculiarities of this infrastructure, it is thus necessary to develop the middleware and the frameworks needed to implement and host the services of an intelligent habitat.

A framework is a set of tools and components which contribute to the development of an application for a given context. A framework is not just a simple library of components but it structures deeply the application. Here frameworks are used to conceive and establish situated services. The 
required frameworks foster the composition of services and devices dynamically. They also provide means to describe and structure distributed reasoning algorithms, especially by to reify the information flow. In addition, they provide basis for the personalization of man-machine interactions by integrating ontologies and profiles construction, for instance to determine habits of living of the elder.

Generally speaking, middleware connects two sides of an application and passes data between them. In the present case, the middleware provides the necessary elements to deploy and access situated services within the smart house. Middleware deals with services connection, service assemblage and service delivery on any device. It gives the necessary anchorage to support code mobility. It addresses issues related to the access to heterogeneous resources, in particular the multimodal access [1]. It dynamically generates a custom user interface for each type of device. Finally it gives access to ontologies which clarify the semantics of devices, services and contents.

\section{The interpretation and decision making layer}

The applications stand at the upper layer. They are most of the time built as extensions of the frameworks and they rely on the middleware. The application layer is composed of the remote monitoring module and the cognitive assistance to the task module. These modules carry out in an autonomous way the interventions toward

- the elder himself, in order to help him to carry out his activities of the everyday life in full safety,

- outside caregivers, medical staff or close relatives, in order to inform them about an imminent danger or the evolution of the incapacities related to the disease.

In order to intervene in an adequate and personalized way, the application layer is made of a supervisor module exploiting information gathered in three models - model of the person, model of the activities and model of the environment. Each model is composed of a metamodel and an instantiation of this metamodel. At the metalevel the concepts used to define a model are described. With that kind of knowledge, the system is able to achieve highlevel reasoning about the concepts. For example, if a person suffers of high blood pressure, and given a definition this disorder, the system can detect when a particular blood pressure value is normal for a person while it could be extremely worrying for another one. Next sections will detail these models and how they are used for reasoning and acting by the supervisor module. 


\section{The model of the person}

The model of the person is divided into three sub-models.

\subsection{The behavioural model}

This model is related to the person's way of life. It contains a description of the person's living habits in terms of the activities performed at particular periods of time. As in [2], this model is organized into several levels of granularity to describe activities: movements, actions, activities of Daily Living (ADL), and living habits.

Movements are directly inferred from raw data acquired from the sensors. These data are filtered, sampled, and organized to become temporal sequences of movements representing for instance the use of the bathroom door, the position of the patient, the occurrence of falls, etc.

Actions are extracted from the most relevant sequences of movements. These sequences are classified into meaningful groups regarding the study of the patient's behaviour. The idea is to get a finite number of types of data - actions or groups - observed along time series, each of them being associated with a finite number of possible symbolic values- the classes.

Activities of Daily Living result from regularities in time occurrence of temporal sequences of actions. They set relevant parameter(s) - activities - that characterize the daily activities of elders at home.

Living habits are obtained from daily observation of the sequences of activities - or parameters of activity - compared with a usual behavioural pattern built from learning in terms of frequency, intensity, duration, time, and/or distribution or order of activities for instance.

\subsection{The physiological model}

This model is dedicated to the person's critical physiological parameters such as blood pressure or heart beat at a given moment. Its content depends on the kind of disease the person is suffering.

\subsection{The cognitive model}

The overall cognitive model results from the integration of the model Act-r [4], Miace [5] and the model of Norman and Shallice [8].

A computational model that would predict the behaviour of a person suffering from the cognitive deficits must take into account formal descriptions of the cognitive capacities of the person. Currently the Act-r 
model is undoubtedly the one that best predicts the behaviour of a normal person [4].

The cognitive model also relies on Miace, a theoretical and dataprocessing architecture dedicated to learning in the case of scientific domains [5]. Miace has the same cognitive architecture principles as Act-r but in addition it takes into account the learning environment and the concept of episodic knowledge. Although Miace was developed within the framework of intelligent tutoring systems, there is a narrow analogy between a person loosing its autonomy and a student: both need a personalized help to achieve a task and this help requires a good knowledge of the cognitive capacities of the person and its mastered skills.

Finally, the intrinsic parallelism in performing the tasks of the everyday life which is hardly managed by people losing cognitive abilities. The Norman and Shallice's cognitive model of the working memory enables to take into account this parallelism. It represents how attention is divided between several activities. That is why it is also integrated in the meta-model of the person [8].

\section{The task model}

The task model contains successful and failed scripts of ADL to be compared to the one performed by the person. ADL are broken down into actions and movements. Failed scripts describe pre-selected erroneous situations due to cognitive impairments. Interventions of the environment can be linked to failed scripts in order to help the person.

\section{The environment model}

The environment model is an instantiation of a metamodel containing generic description of equipments and habitat: categories of rooms, residential furniture, sensors, electrical appliances... However the generic description cannot know beforehand how to perform an elementary task with any particular device. For example, the knowledge of which button should be used to set microwave temperature is part of the instantiated environment model. This kind of information is provided directly from reflective features of the specific microwave used in the concrete environment.

\section{The supervisor module}

The decision-making process is split between the two application modules, that is the telemonitoring module $(\S 8.1)$ and the task-support module for 
cognitive assistance ( $\$ 8.2)$. These two modules exploit information available in the three models on person, activities and environment. These modules use the models differently and for different purposes. The telemonitoring module bases its diagnosis process on "simple" patterns of numeric threshold values that triggers external actions sent outside the habitat. In contrast cognitive assistance perform complex symbolic diagnosis that triggers local actions performed inside the habitat.

\subsection{The telemonitoring module}

The telemonitoring module carries out the analysis of the behavioural patterns in order to detect abnormal behaviours of the monitored person. It detects patterns of simple events associated to a risk. The combination of these events are likely to deteriorate the mental and physical integrity of the person. Obviously the implementation is centred on mechanisms germane to pattern matching. This mechanism must be as accurate as possible, noise resistant and able to adapt to failures or absence of sensors. Intervention strategies will suggest how to reduce or to mitigate the potentially risky behaviours, for instance by physical reinforcement or by changing the environment.

Various mathematical methods and logics can be used to achieve these various tasks. On the mathematical level, according to the nature of the problem, these methods extend from multi-factorial analysis, principal components analysis or neural networks to hidden Markov models or bayesian networks. On the logical level, decision-making relies on various traditional strategies and knowledge representation used in artificial intelligence (inference in first order logic or in description logic). These techniques which were developed and used for the textual data processing will be transposed and adapted to the context of this project. They are all integrated in the SATIM system [6].

\subsection{The cognitive assistance module}

The task support module transforms and progressively integrates raw data coming from the sensors into a plan representing the intentions of the person. Then the model of Rousseau [13] takes the three models person, activities and environment and diagnose if the elder is in a competence situation or in a handicap one, establishing if the current situation requires an intervention and if yes, which one would be appropriate.. This model was conceived specifically for home adaptation. 


\section{Implementation}

To performs their duties, algorithms are partly local and partly distributed. Indeed one of the issues is transform intrinsically centralized models and reasoning algorithms into decentralized ones. By decentralizing them, decisions are made closer to the components on which they act. This approach renders the system more tolerant to the faults. Epitalk [3] is a platform to develop such support systems. Epitalk enables to process data on a hierarchical and distributed basis. It was applied for plan recognition. At the lowest level analysis functions will be integrated in intelligent sensors and actuators to carry out the transformation of the raw data into symbols. The result of these analyses will be transmitted to higher nodes which will have a broader perspective on the environment and the realization of the task. As a consequence, the decision-making centres will be located as close as possible to the places where their decisions take effect.

\section{Conclusion}

This paper has sketched a pervasive infrastructure and applications that can extend the period the elders may continue to live safely in their houses, even if they are suffering from cognitive disease. It is a combination of cognitive assistance offering immediate local support and telemonitoring alerting external relatives and caregivers in case of emergency. Models of person, activities and environment are available to personalize the support provided. The implementation is based on pervasive computing, spontaneous networking and distributed systems. It offers the possibility of a constant reorganization devices, processes and algorithms. A middleware and many frameworks provide flexible links between devices and applications. The new reality can be seen here as a symbiosis between the human being and the machine with an aim of allowing a better realization of the human tasks.

\section{References}

[1] Carboni, D; Piras, A.; Sanna, S.; Paddeu G. (2002) On-the-fly Generation of UIs for Ubiquitous Applications UIST'02 User Interface Software \&Technology, Oct.27-30, 2002. Paris, France.

[2] Duchêne, F., V. Rialle et N. Noury (2002). Télésurveillance médicale à domicile : proposition d'une architecture pour un système de détection de situations critiques et de décisions sur l'état du patient. Neuvièmes Journées Francophones d'Informatique Médicale (JFIM), Québec, May 6-7, 2002, Springer-Verlag. 
[3] Giroux, S., Paquette, G. Girard, J., (1996) Epiphyte Advisor Systems For Collaborative Learning, CALISCE '96, Donostia - San Sebastián, July 29-31, 1996, Springer LNCS 1108, pp. 42-50.

[4] Glasspool, D. W., Cooper, R. (2002). Executive Processes. In R. Cooper (Ed.) Modelling High Level Cognitive Processes. New Jersey: Lawrence Erlbaum Associates. pp. 313-362.

[5] Mayers A., Lefebvre B \& Frasson C. (2001). Miace: A Human Cognitive Architecture. SIGCUE outlook. 27 (2) pp. 61-77.

[6] Meunier, J.G., Biskri, I., SATIM : une plate-forme modulaire pour la construction de chaînes d'analyse de textes assistée par ordinateur. to appear in Arnould, J.C., Blum, C. (Eds). L'édition électronique : état des lieux. Presses de L'Université de Rouen, 22 pages.

[7] Ministère de la Santé et des Services sociaux. Direction générale de la planification et de l'évaluation. (1998). Le virage ambulatoire en santé physique: Enjeux et perspectives. Coll. Études et analyses. Québec.

[8] Norman, D.A., Shallice, T. (1986). Attention to action: Willed and Automatic control of behavior. In R.J. Davidson, G.E. Schwarts, \& D. Shapiro (Eds), Consciousness and self-regulation. Advances in research and theory, Vol. 4, New-York: Plenum Press. pp. 1-18.

[9] Pigot H., Mayers A., Giroux S., Lefebvre B., Rialle V., Noury N., (2002) Smart house for frail and cognitive impaired elders, UbiCog '02: First International Workshop on Ubiquitous Computing for Cognitive Aids, Göteborg, Sweden, September 29th, 2002.

[10] Rialle, V., F. Duchêne, N. Noury, L. Bajolle et J. Demongeot (2002). Health 'smart' home: Information technology for patients at home. Telemedicine Journal and E-Health. 8 (4), (in press).

[11] Rialle .V, Noury N., Hervé T. (2001). An experimental Health Smart Home and its distributed Internet-based Information and Communication System : first steps of a research project. In V. Patel et al. (eds): MEDINFO 2001, 10th World Congress on Medical Informatics. Amsterdam: IOPress. pp. 1479-1483.

[12] Roberts, D., Johnson, R. (1997) Evolving Frameworks A Pattern Language for Developing Object-Oriented Frameworks, in R. Martin, et al. eds, Pattern Languages of Program Design, vol.3, AddisonWesley, 1997.

[13] Rousseau, J., Potvin, L., Dutil, É., Falta, P. (in press). Model of Competence: A Conceptual Framework for understanding the PersonEnvironment Interaction for Persons with motor disabilities. Journal of Occupational Therapy in health Care. 